\title{
El desarrollo de la escritura analítica: aprender a enlazar la información, aprender a posicionarse
}

\author{
Developing Analytical Writing: Learning to Connect \\ Information, Learning to Take a Stance \\ Elisa Rosado Villegas ${ }^{1}$, Iban Mañas Navarrete ${ }^{1}$, \\ Irene Yúfera Gómez ${ }^{1}$ y Melina Aparici Aznar ${ }^{2}$
}

${ }^{1}$ Universitat de Barcelona

${ }^{2}$ Universitat Autònoma de Barcelona

\section{Resumen}

\begin{abstract}
Estudiantes de todos los niveles muestran problemas con ciertas convenciones del discurso escrito consideradas indicadoras de desarrollo y predictoras de la calidad de los textos: la organización de la información y el uso de recursos específicos. El uso de conectores y marcadores discursivos contribuye a la cohesión del texto y guía la interpretación de las relaciones entre sus componentes. Analizamos los textos analíticos en catalán de 128 alumnos de secundaria y universitarios para identificar las dificultades y las trayectorias evolutivas en la conexión intra e interoracional. Los textos fueron producidos tras aplicar una secuencia didáctica sobre escritura académica. Examinamos el repertorio de marcas de conexión (conjunciones, marcadores parentéticos, marcadores léxicos) y las funciones que desempeñan (proposicional, estructural, modal). Las diferencias entre grupos aparecen 1) en el uso de conjunciones, más empleadas en secundaria, y marcadores parentéticos, en universitarios, y 2) en las funciones de marcadores modales que expresan el posicionamiento del autor. La coherencia de los textos depende del uso de marcas de conexión, su función en la estructura textual y el posicionamiento del autor. Aunque las marcas de conexión interoracional de adolescentes y adultos son escasas, la expresión del posicionamiento discursivo por medio de marcadores modales sí evoluciona.
\end{abstract}

Palabrasclave: escritura analítica, conexión intra e interoracional, conectores, marcadores del discurso, desarrollos posteriores del lenguaje

Correspondencia a:

Elisa Rosado Villegas

Departament d'Educació Lingüística i Literària, Facultat d'Educació, Campus Mundet,

Edifici Llevant, Pg. Vall d'Hebron, 17108035 Barcelona, España

erosado@ub.edu

(C) 2021 PEL, http://www.pensamientoeducativo.org - http://www.pel.cl

ISSN:0719-0409 DDI:203.262, Santiago, Chile doi: 10.7764/PEL.58.2.2021.10 


\begin{abstract}
The development of analytical writing requires mastery of specialized linguistic forms and functions. Students of all educational levels have problems with the organization of information and the use of specific formal resources. We focus on intra-sentential connectives and discourse markers that contribute to text cohesion and guide the logicalsemantic relationships between units of discourse, as they are considered predictors of writing development and indicators of text quality. We analyze the texts produced by 128 Catalan high school and university students in order to identify the difficulties they have in establishing connectivity and defining developmental trajectories. The texts were produced after implementing a didactic sequence on academic writing. We examine the repertoire of markers (conjunctions, parenthetical markers, lexical markers) and the discursive functions they perform (propositional, structural, modal). The results show differences between the two groups in the use of conjunctions, which are used more frequently by high school students, and parenthetical markers, which are more commonly used by university students. With regard to discourse functions, differences between the groups also appear in modal markers. The structural efficacy and coherence of a text are related to proficient use of connectivity markers, their function within a text, and the expression of the writer's stance. The use of structural markers is uncommon for both groups, but university students are more willing to use them in order to express their stance.
\end{abstract}

Keywords: analytical writing, intra- \& inter-sentential connectivity, connectors, discourse markers, later language developments

\title{
Introducción
}

En su incorporación a la vida académica, los alumnos deben familiarizarse con las convenciones de la escritura analítica, es decir, el lenguaje de los textos producidos en contextos académicos (Schleppegrell, 2004; Snow \& Uccelli, 2009). El término 'escritura analítica' refiere típicamente a textos centrados en el análisis y la argumentación (Schleppegrell, 2004; Uccelli, Deng, Phillips Galloway \& Qin 2019), con componentes tanto expositivos como argumentativos, que requieren de un uso apropiado de formas y funciones lingüísticas específicas (Ruiz Bikandi \& Tusón, 2002 $)^{1}$ y cuya calidad y efectividad tiene consecuencias sociales y académicas directas en las posibilidades de acceso a la educación superior y en la promoción en ámbitos profesionales cualificados (Uccelli et al., 2015). En comparación con géneros discursivos cognitivamente menos complejos y evolutivamente más tempranos, como la narración, los analíticos se caracterizan por la necesidad del autor de expresar su punto de vista sobre un tema, presentar argumentos a favor y en contra y ofrecer evidencia adecuada o posibles refutaciones (Toulmin, 2002). El resultado esperado es que el lector comparta el punto de vista del autor, persuadido por la solidez de su argumentación (Driver, Newton \& Osborne, 2000).

La producción de este tipo de textos constituye una preocupación constante para profesores de todos los niveles y disciplinas por cuanto, si bien se introducen en la educación primaria - en el sistema educativo español- y se trabajan y tratan específicamente en formación secundaria obligatoria y preuniversitaria, presentan para el alumnado dificultades que perduran en su tránsito a la universidad e incluso más allá. Diversos estudios evolutivos

1. El artículo recoge resultados del proyecto de investigación Posicionament i Informació (PI): La forma i el fons dels textos analitics en educació secundària i educació superior. IP.: E. Rosado (PI-REDICE-18-1920; Institut de Desenvolupament Professional - Institut de Ciències de l'Educació, Universitat de Barcelona). 
muestran que las dificultades para escribir textos argumentativos eficaces no se acaban con la incorporación a la universidad (Bañales, Vega, Araujo, Reyna \& Rodríguez, 2015; Carlino, 2005; Castelló, 2007). De hecho, no son pocos los estudiantes de postgrado e investigadores noveles que encuentran dificultades en la redacción de sus trabajos académicos e informes científicos (Gavari \& Tenca, 2017).

Algunos de los problemas que tradicionalmente detectan profesores de todos los niveles educativos en los textos de sus alumnos están relacionados 1) con el aprendizaje de convenciones del discurso escrito relacionadas con la organización de la información y el uso competente de las formas y funciones lingüísticas características de este tipo de textos, y 2) "con un uso flexible de un repertorio de formas léxico-gramaticales y discursivas de desarrollo tardío para organizar las ideas y expresar un posicionamiento en diferentes textos académicos" (Uccelli, Dobbs $\&$ Scott, 2013, p. 39). Adoptar una postura discursiva clara y materializar una argumentación supone que el autor ha desarrollado un pensamiento crítico que le permite posicionarse ante temas específicos, pero, además, este debe reconocer y apropiarse de los recursos que su lengua le ofrece para argumentar a favor o en contra de un asunto. En los textos analíticos se espera que los autores sean capaces de emplear un vocabulario diverso, pero preciso, de condensar la información mediante, por ejemplo, una sintaxis compleja o nominalizaciones y, asimismo, de explicitar la organización del discurso mediante marcas de conexión intra e interoracional, que indiquen no solo las transiciones textuales sino también el posicionamiento del autor (Aparici, Cuberos, Salas \& Rosado, 2021; Schleppegrell, 2004; Snow \& Uccelli, 2009).

Las marcas de conexión interoracional señalan las relaciones que se establecen entre componentes del discurso mayores que la oración (Aparici, Rosado \& Salas, 2016; Aparici et al., 2021; Choi, 2007) y la capacidad de explicitar dichas relaciones por medio de estos elementos, marcadores discursivos, es un desarrollo evolutivo tardío (Katzenberger \& Cahana-Amitay, 2002). Por su parte, los conectores intraoracionales contribuyen a facilitar la extracción de relaciones entre cláusulas y también constituyen un desarrollo tardío (Aparici, 2010; Rosado, Aparici \& Perera, 2014).

El uso de mecanismos explícitos de conexión (por ejemplo, conjunciones en oraciones coordinadas y subordinadas o marcadores discursivos en la conexión interoracional) constituye un logro cognitivo complejo, relativamente tardío en el proceso de adquisición del lenguaje (Aparici, 2010; Berman, 1988), pero, además, su desarrollo está directamente influido por el género discursivo de la misma manera que lo está el desarrollo de la producción textual en general (véase Aparici, 2010; Berman, 2008; Rosado et al., 2014, Aparici, Rosado \& Oliver, 2014). Así, las características relacionadas con la complejidad sintáctica varían entre géneros y, por ejemplo, mientras el uso de sintagmas nominales complejos se localiza preferentemente en los géneros analíticos típicos de contextos académicos, la subordinación que va más allá de la modificación de estos sintagmas es más típica de otros géneros (Biber \& Gray, 2010; Biber, Gray, Staples \& Egbert, 2020). Sin embargo, en la mayoría de los estudios sobre desarrollo del discurso, la subordinación, entendida como medida de complejidad gramatical, se interpreta como signo de madurez, característica de géneros no narrativos (por ejemplo, expositivos o argumentativos). Estudios como el de Aparici et al. (2016) o el de Cuberos (2019) atestiguan tanto la influencia del género discursivo en el uso de marcadores discursivos como la relevancia de estas formas en géneros con un componente expositivo como los que nos ocupan (Montolío, 2001).

Tanto los marcadores discursivos como la complejidad sintáctica realizada por medio del uso de conectores intraoracionales aportan una información valiosa en la evaluación de un texto. Su uso determina la percepción de la calidad de su estructura, de la solidez y eficacia de los argumentos, de los razonamientos y conclusiones expuestos o, de forma determinante, de las relaciones lógico-semánticas que se establecen entre las partes del discurso. Por definición, conectores sintácticos y marcadores discursivos contribuyen a la cohesión textual y funcionan como guía en la interpretación de las relaciones entre las partes del texto (Givón, 1992; Hyland, 2002). 
En trabajos previos que consideran separadamente el uso de conectores intraoracionales y marcadores interoracionales, se han identificado patrones de desarrollo diferentes para ambos tipos de elementos (Aparici, 2010; Tolchinsky, Aparici \& Salas, 2011; Aparici et al., 2014; Rosado, Aparici, Horcada, Cuberos \& Salas, 2017). Sin embargo, estudios recientes que consideran conjuntamente marcas de conexión intra e interoracionales atendiendo a criterios eminentemente funcionales encuentran resultados que apuntan a las ventajas de emplear criterios que van más allá de lo formal. Siguiendo el enfoque de Cuenca (2006, 2013), en el trabajo de Gras, Galiana y Rosado (2020) se compara el uso que realizan hablantes nativos y no nativos de espańol de marcas de conexión intra e interoracionales clasificadas en tres macrofunciones discursivas: proposicional, estructural y modal (véase el apartado Categorías y criterios de análisis). Según los resultados, las diferencias de uso entre sus dos poblaciones vienen determinadas por la interacción entre el rol sintáctico o discursivo de estas formas y la naturaleza de la función que desempeñan. Si bien en el caso de marcadores con función proposicional apenas se observan diferencias, en el uso de los conectores modales y estructurales los hablantes no nativos presentan más problemas para asignar los valores discursivos apropiados.

En trabajos anteriores, además de posibles cambios evolutivos, hemos tratado de detectar cómo estudiantes de distintos niveles educativos son sensibles al trabajo pedagógico específico sobre las características de la escritura analítica (Aparici et al., 2021). En relación con el uso de marcadores, los resultados indican que un análisis de marcadores discursivos que prescinda de criterios semánticos o funcionales puede explicar en parte que no se observen diferencias relevantes: la de los marcadores resulta una categoría 'paragua' demasiado amplia, incapaz de capturar los múltiples matices de estos elementos.

Sin embargo, cuando se atiende a criterios semántico-funcionales, como en el trabajo de Aparici y sus colegas (2014) sobre textos orales y escritos, los resultados permiten identificar patrones evolutivos y, además, revelan un efecto de la modalidad de producción que sugiere que en los textos escritos la cohesión descansa en mayor medida que en los orales en otros recursos lingüísticos como, por ejemplo, los conectores sintácticos.

Como vemos, y a partir de resultados como los aquí descritos, se acentúa la conveniencia de emplear, además de criterios formales al uso, criterios funcionales capaces de revelar cambios evolutivos en la interfaz sintaxisdiscurso (Brandes \& Ravid, 2019) y disponer, así, de una perspectiva más completa y ajustada.

En este estudio examinamos cómo se conecta la información proporcionada en los textos analíticos de estudiantes de educación secundaria y universitarios. Por una parte, nos interesa describir el uso de los elementos de conexión que operan a nivel oracional y a nivel discursivo (conexión intra e interoracional); por otra, queremos identificar el modo en que estas formas desempeñan funciones discursivas específicas cuando proporcionan argumentos, evidencias y ejemplos con objeto de convencer al lector potencial de la validez de su argumentación. Para ello, los textos analíticos fueron recogidos en un contexto pedagógicamente controlado y ecológicamente válido; la aplicación de la misma secuencia didáctica y el empleo de las mismas actividades y materiales en los dos grupos de participantes permite 1) controlar explícitamente el input que estos reciben y 2) disponer de un corpus comparable en los dos grupos de edad.

Así, con el objetivo de describir y tipificar el modo en el que se realiza la conexión de la información en los textos analíticos escritos por alumnos de secundaria y de educación superior, nos propusimos 1) identificar los usos de marcas de conexión textual empleados por los participantes en los dos grupos de edad a nivel intraoracional e interoracional (formas) y 2) describir cómo se introduce y relaciona la información mediante el establecimiento de relaciones lógico-semánticas específicas (funciones). 
Dado que en estos contextos aún no se dispone de herramientas validadas para una evaluación específica de la calidad de los textos analíticos, esperamos que nuestros resultados proporcionen información sobre cuáles son las marcas de conexión más problemáticas para alumnos de niveles diferentes que, por consiguiente, merecen una atención especial en el diseño de herramientas de evaluación pedagógicamente informativas (Uccelli, Dobbs \& Scott, 2013).

\section{Método}

\section{Participantes}

En el estudio participaron 128 estudiantes de dos niveles educativos: 4o de Educación Secundaria Obligatoria (ESO) y primeros cursos de estudios universitarios. Los participantes vivían en Barcelona o alrededores y pertenecían a seis grupos-clase de cuatro escuelas y dos grupos-clase de una universidad. La muestra estaba conformada por 72 (34 mujeres, 38 hombres) estudiantes de secundaria $(M=15,80$ años $[18,00 ; 15,10])$ y $56(42$ mujeres, 14 hombres) estudiantes universitarios $(M=20,01$ años [24,80;19.10]). No se excluyó a ningún participante por su bagaje sociolingüístico, y ninguno presentaba dificultades de aprendizaje o comportamiento conocidas. ${ }^{2}$

Dada la importancia de las variables sociodemográficas y sociolingüísticas en el desarrollo y producción de la escritura, recogimos esta información mediante un cuestionario ${ }^{3}$. La lengua en que se recogieron los textos de los participantes es el catalán, que es además lengua de la escuela en nuestro contexto (Cataluña, España); mediante el cuestionario sociolingüístico administrado comprobamos que todos los participantes son en algún grado bilingües de catalán y español (Tolchinsky, Aparici \& Vilar, 2021). ${ }^{4}$

\section{Materiales y procedimiento}

Los textos analizados fueron producidos tras la aplicación de una secuencia didáctica diseñada para sensibilizar a los participantes sobre las características de la escritura académica (Aparici et al., 2021). A lo largo de siete sesiones, los participantes realizaron durante sus clases habituales un conjunto de actividades con su profesor, que había recibido formación sobre la implementación de las actividades y la ejecución de las tareas respetando el diseño del estudio para garantizar un mismo contexto pedagógico para todos los grupos. Estas actividades incluían lecturas sobre el tema tratado, discusiones en clase, producción de textos analíticos y evaluación de fragmentos de textos de compañeros a partir de una rúbrica, así como la reflexión sobre aspectos clave de estos textos como la adopción de distintas posiciones enunciativas: de autor, de lector y de evaluador (para mayor detalle, véase Alonso-Cortés, Llamazares \& Zapico, 2017).

2. El corpus fue recogido en el marco del proyecto ESCANDILING Escritura analitica y diversidad lingüistica: Cambios evolutivos y microevolutivos desde la educación primaria a la educación superior. IP.: L. Tolchinsky, J. Perera. (EDU201565980-R; Ministerio de Economía y Competitividad).

3. La información sobre la experiencia lingüística de los participantes se recoge por medio del MUAQ (Multilingual Use Assessment Questionnaire), cuestionario para la evaluación del multilingüismo desarrollado en el contexto del proyecto ESCANDILING para obtener información sobre aspectos sociolingüísticos, socioeconómicos y de hábitos culturales generales de hablantes multilingües (Aparici \& García-Palau, 2019). El cuestionario hace hincapié en los usos lingüísticos en el ámbito familiar, escolar y social de este tipo de poblaciones.

4. Se obtuvo el consentimiento informado de los participantes o de sus tutores en el caso de tratarse de menores, de acuerdo con los procedimientos especificados para los proyectos de investigación en la normativa de la UB recogida en su Código de buenas prácticas en investigación (http://diposit.ub.edu/dspace/bitstream/2445/28543/1/codibonespractiques_spa.pdf) 
Durante estas sesiones, cada uno de los participantes produjo cinco textos: el texto 1 sobre el tema A (libertad de vestir), antes del comienzo de la secuencia didáctica; los textos 2, 3 y 4 sobre el tema B (libertad de movimiento entre países), respectivamente antes, durante e inmediatamente después de las actividades de la secuencia, y el texto 5 sobre el tema C (premios y castigos), un mes después de finalizar las actividades. En este estudio se analiza el texto 4.

Los textos fueron producidos por los estudiantes en ordenador, accediendo a un formulario de Google Forms en el que quedaban registrados. Todos los textos (128) fueron transcritos y codificados para su análisis en formato CHAT (proyecto CHILDES; MacWhinney, 2000), y los datos se analizaron utilizando programas CLAN (proyecto CHILDES). Los textos fueron segmentados en cláusulas como unidad básica de análisis, siguiendo las indicaciones de Berman y Slobin (1994).

\section{Categorías y criterios de análisis}

La herramienta que empleamos para el análisis de las marcas de conexión textual es una taxonomía según la cual estos elementos constituyen una clase funcional que incluye diferentes tipos de palabras, y que considera tanto formas léxicas como funciones discursivas. Validada inicialmente para el catalán, y confeccionada a partir de la propuesta de Gras et al. (2020), esta taxonomía se define según dos criterios. ${ }^{5}$ Atendiendo a criterios estrictamente formales, identificamos 1) conjunciones coordinantes y subordinantes que conectan unidades en el nivel oracional, 2) marcadores parentéticos, unidades léxicas fijas que constituyen una clase cerrada y que coinciden mayoritariamente con los marcadores del discurso tradicionales (Martín Zorraquino \& Portolés, 1999), y 3) marcadores léxicos, que operan también en el nivel de discurso, pero constituyen un repertorio abierto de elementos que presentan un grado variable de gramaticalización (Cuenca, 2017); prototípicamente metadiscursivos, suelen estar constituidos por una cláusula que mantiene su significado composicional y pueden sustituirse por un conector gramatical parentético.

Por otra parte, desde una perspectiva funcional, distinguimos tres tipos de marcadores: 1) proposicionales, típicamente conjunciones que conectan oraciones compuestas y que articulan contenidos como adición, contraste, temporalidad, causa, consecuencia, etc., 2) estructurales, que señalan la demarcación de unidades textuales e implican generalmente un avance discursivo, por ejemplo, en la trama de la narración o en la estructura de la argumentación, en aperturas o cierres, en reorientaciones o reformulaciones, en la continuación de un tema o argumento, etc., y 3) modales, que expresan el posicionamiento del autor y típicamente muestran el vínculo entre los interlocutores o la actitud del hablante que con ellos expresa duda, justificaciones, opinión, énfasis, etc.

Todas las marcas de conexión se clasificaron según los dos criterios. Para garantizar la idoneidad de la herramienta, se realizó un análisis de fiabilidad entre jueces para la identificación y categorización de marcas de conexión textual (cuatro jueces) para 20\% de los textos. Los resultados del coeficiente de correlación intraclase (consistencia interna) indicaron un alto grado de fiabilidad entre jueces (.845) con un 95\% de intervalo de confianza (IC) [.21, .98].

\section{Análisis de datos}

Tras la codificación de los marcadores, se realizó un contraste de medias de muestras independientes sobre el total de marcas de conexión para comparar su uso entre los niveles educativos. Los análisis por forma y por función de los marcadores se llevaron a cabo de manera independiente. Debido a que los datos no se ajustaban a las condiciones de aplicación de normalidad e igualdad de varianzas, se aplicó un análisis de varianza de

5. La herramienta se desarrolló y validó en el marco del proyecto Posicionament i Informació (PI): La forma i el fons dels textos analitics en educació secundària i educació superior. IP.: E. Rosado (PI-REDICE-18-1920; Institut de Desenvolupament Professional - Institut de Ciències de l'Educació, Universitat de Barcelona). 
rangos de Friedman mixto con nivel educativo como variable intersujeto y tipo de marcador como variable intrasujeto. Todos los análisis se realizaron en el entorno computacional R (RStudio Team, versión 3.5.26), utilizando los paquetes ARTools (Wobbrock, Findlater, Gergle \& Higgins, 2011) y nparcomp (Konietschke, Placzek, Schaarschmidt \& Hothorn, 2015).

\section{Resultados}

Para ofrecer una caracterización de los textos más allá del análisis de las marcas de conexión objeto de estudio y situar la magnitud de uso de dichos elementos, se presentan en primer lugar medidas referidas a la extensión de los textos producidos. En la tabla 1 se recogen los descriptores de estas medidas: número total de palabras y cláusulas, total de marcas de conexión textual y proporción que estas suponen sobre el total de palabras de los textos, para cada nivel educativo.

Tabla 1

Extensión de los textos y uso de marcadores. Secundaria y universidad (media y DT)

\begin{tabular}{ccc|cc|ccc|cc} 
& \multicolumn{4}{c}{ Extensión } & \multicolumn{4}{c}{ Marcas de conexión } \\
\cline { 2 - 10 } Nivel educativo & \multicolumn{2}{c|}{ No de palabras } & \multicolumn{2}{c|}{$\begin{array}{c}\text { No de } \\
\text { cláusulas }\end{array}$} & \multicolumn{2}{c}{$\begin{array}{c}\text { No de } \\
\text { marcadores }\end{array}$} & \multicolumn{2}{c}{$\begin{array}{c}\text { No de marcas/ } \\
\text { No de palabras }\end{array}$} \\
\cline { 2 - 11 } & M & DT & M & DT & M & DT & M & DT \\
\hline Secundaria (ESO) & 238,16 & 74,42 & 20,65 & 16,19 & 11,22 & 4,33 & 4,83 & 1,64 \\
\hline Universidad (UNI) & 367,66 & 109,99 & 33,80 & 24,23 & 12,78 & 6,68 & 3,48 & 1,45 \\
\hline
\end{tabular}

Fuente: Elaboración propia.

Respecto de la extensión de los textos, observamos que, tanto en número de palabras como en número de cláusulas, los textos producidos por el grupo UNI se caracterizan en promedio por una mayor extensión en comparación con el grupo ESO, así como por una variabilidad más alta para los dos indicadores de extensión. La comparación de medias para dos muestras independientes con la corrección de Welch entre ambos grupos confirmó que el grupo UNI produce textos significativamente más largos, en número total de palabras, que el grupo $\operatorname{ESO}(t=-7,564, g l=92,126, p<0,001, d=1,41$, IC 95\% [-163,49; $-95,49])$, con una diferencia mayor que una desviación estándar. Los resultados de la misma prueba sobre el número total de cláusulas confirman esta diferencia significativa $(t=-3,498, g l=91,323, p<0,001, d=0,65$, IC 95\% $[-20,61 ;-5,68])$, en este caso con un tamaño del efecto medio-alto. Por otro lado, observando las columnas $n^{\circ}$ de cláusulas y $n^{\circ}$ de marcadores, vemos que en promedio los elementos de conexión están presentes en la mitad (ESO) y un tercio (UNI) de las cláusulas de un texto (véase la tabla 1), lo que indica que se trata de elementos de uso frecuente.

Dado que los participantes produjeron textos de diferente extensión, los análisis sobre el uso de marcas de conexión se realizaron a partir de las medias de las proporciones de conectores-marcadores sobre el número total de palabras de cada texto. Si bien los textos de los estudiantes universitarios contienen un número total de marcadores superior al de los textos del grupo de secundaria, después de ponderar el uso total de marcadores por la longitud del texto se observa que la relación entre los dos grupos se invierte, de modo que realmente la

6. www.rstudio.com 
frecuencia de uso de estos elementos en los textos de ESO es mayor que en los de UNI. La comparación de las medias de las proporciones confirma que los alumnos de secundaria presentan una proporción significativamente mayor de marcadores, con un tamaño del efecto alto $(t=4,924, g l=123,84, p<0,001, d=0,86$, IC 95\% [0,81; $1,90]$ ); esto sugiere una profusión de uso de marcadores en la escritura de textos analíticos entre los estudiantes de secundaria que se reajusta en etapas educativas posteriores. Los resultados sobre forma y función de los marcadores identificados en los textos nos permitirán concretar en qué tipo de marcadores se da este reajuste entre los dos grupos de participantes. A continuación, presentamos los resultados de ambos análisis.

Para analizar el uso de los diferentes tipos de conectores intra e interoracionales según su forma, se computó la proporción de conjunciones, marcadores parentéticos y marcadores léxicos sobre el número total de marcadores en cada texto y se obtuvo su distribución. En la tabla 2 se muestra un resumen de los descriptivos de esta distribución para los dos grupos de edad. Mientras que en los textos de secundaria las conjunciones son el tipo de marcador más frecuente, los textos de los universitarios presentan una mayor proporción de parentéticos que de los otros tipos de marcadores. La presencia de marcadores léxicos es la más baja en ambos grupos. A su vez, las conjunciones son más frecuentes en ESO que en UNI, y con los marcadores parentéticos ocurre a la inversa.

Tabla 2

Distribución de la proporción de marcadores según su forma. Secundaria y universidad (media y DT)

\begin{tabular}{ccc|ccc|cc} 
& \multicolumn{6}{c|}{ Tipo de marcador } \\
\cline { 2 - 7 } Nivel educativo & \multicolumn{2}{c}{ Conjunciones } & \multicolumn{2}{c}{ Parentéticos } & \multicolumn{2}{c}{ Léxicos } \\
\cline { 2 - 7 } & $\mathrm{M}$ & DT & M & DT & M & DT \\
\hline Secundaria (ESO) & 54,44 & 19,29 & 31,26 & 18,69 & 14,27 & 12,69 \\
\hline Universidad (UNI) & 38,92 & 19,47 & 48,55 & 17,99 & 12,52 & 11,92 \\
\hline
\end{tabular}

Fuente: Elaboración propia.

Para confirmar la existencia de diferencias significativas en las distribuciones entre los dos grupos, comprobamos las condiciones de aplicación del análisis de varianza mixto, con la variable de grupo (ESO y UNI) como variable intersujeto y el tipo de elemento de conexión como variable intrasujeto. Dado que el resultado de la prueba de Levene no permitió asumir varianzas equivalentes entre los grupos $(F(5,378)=3,78, p=0,002)$, realizamos un ANOVA de rangos de Friedman. Esta prueba resulta más robusta con la suma de cuadrados cuando los datos no son normales y pertenecen a un diseño no balanceado, como en este caso.

Los resultados confirmaron un efecto significativo para el tipo de elemento de conexión $(F(2,21487)=20,43$, $p<0,001, \bigotimes 2=0,44)$ con un tamaño del efecto grande; no se detectó, en cambio, un efecto significativo del nivel educativo $(F(1,378)=0,01, p=0,914, \bigotimes 2<0,01)$. También se confirmó un efecto significativo de la interacción entre tipo de marcador y nivel educativo con un tamaño del efecto mediano $(F(2,378)=32,18, p<0,001$, $\bigotimes 2=$ $0,14)$. Los resultados indican que existen diferencias significativas en las distribuciones de los grupos ESO y UNI en cuanto a los tipos de marcadores según su forma.

Para determinar la naturaleza de esas diferencias, realizamos una serie de comparaciones por pares entre los niveles de las variables intra e intersujeto con la corrección de Bonferroni sobre el nivel $\bigotimes$ de las pruebas de contraste. En el primer conjunto de comparaciones contrastamos las medias de los dos grupos dentro de cada tipo de marcador. 
Los resultados confirmaron una proporción significativamente superior de conjunciones en el grupo ESO sobre el grupo UNI $(t=5,44, p<0,001)$, y una proporción superior de marcadores parentéticos en UNI en comparación con ESO $(t=-5,82, p<0,001)$. El uso de nexos intraoracionales, que establecen relaciones de coordinación o subordinación, es obligatoria en el contexto en el que aparecen. Si, además, la producción de escritores menos expertos incluye la repetición de conjunciones despojadas de su valor original para unir proposiciones, la proporción de conjunciones se dispara. Como vemos en el ejemplo (1), la conjunción $y$ pierde su valor de adición y pasa a funcionar como un marcador interoracional. Este fenómeno, conocido como pegamento discursivo (discourse glue), ha sido atestiguado en etapas más tempranas y es sobre todo típico del discurso oral (Aparici, 2010; Aparici et al., 2016; Berman, 1996).

(1) Trobem notícies cada dia de gent que intenta creuar una frontera i no ho aconsegueix i ho paga amb la vida i això em fa pensar. [Secundaria, 015]

Trad.: Encontramos noticias cada día de gente que intenta cruzar una frontera y no lo consigue y lo paga con la vida y eso me hace pensar.

Paralelamente, y a pesar de la instrucción explícita que supone la implementación de la secuencia didáctica en los dos grupos, son los universitarios los que sienten la necesidad de incorporar marcadores parentéticos para relacionar contenidos y estructurar sus textos de forma explícita como se ilustra en el ejemplo de (2):

(2) Així_doncs, han recollit una sèrie de dades sobre camps de refugiats [Universidad, 18]

Trad.: Así pues, han recogido una serie de datos sobre campos de refugiados.

Por último, tal y como sugerían los datos descriptivos, las proporciones de marcadores léxicos en los textos de ambos grupos son estadísticamente equivalentes $(t=0,96, p=0,333)$; el repertorio de formas incluye ejemplos como los de (3a) y (3b):

(3a) I què me'n dieu dels que moren al mar ofegats? [Secundaria, 01]

Trad.: ¿Y qué me decís de los que mueren en el mar ahogados?

(3b) Estic segur que si aquí, a Europa hi haguessin les guerres que hi ha a Àfrica,

ells estarien encantats d'ajudar-nos i de donar-nos una casa i un treball. [Secundaria, 01]

Trad.: Estoy seguro de que, si aquí en Europa hubiera las guerras que hay en África, ellos estarían encantados de ayudarnos y de darnos una casa y un trabajo.

Con el segundo conjunto de comparaciones exploramos las diferencias entre los tres tipos de marcadores dentro de cada grupo. En cuanto al grupo de secundaria, los resultados indican una proporción significativamente mayor de conjunciones que de marcadores parentéticos $(t=7,28, p<0,001)$ y marcadores léxicos $(t=15,73$, $p<0,001)$; a su vez, la proporción de parentéticos también es significativamente mayor que la de léxicos $(t=$ $6,51, p<0,001)$. Dentro del grupo UNI, la proporción de conjunciones también es significativamente mayor que la de marcadores léxicos $(t=9,06, p<0,001)$, como ocurre en el grupo de secundaria. En cambio, el uso de marcadores parentéticos resulta significativamente superior al de conjunciones $(t=3,03, p=0,003)$ y al de marcadores léxicos $(t=13,54, p<0,001)$. En el gráfico 1 se ilustra el comportamiento del conjunto de datos de los tipos de marcadores según su forma por grupo. 


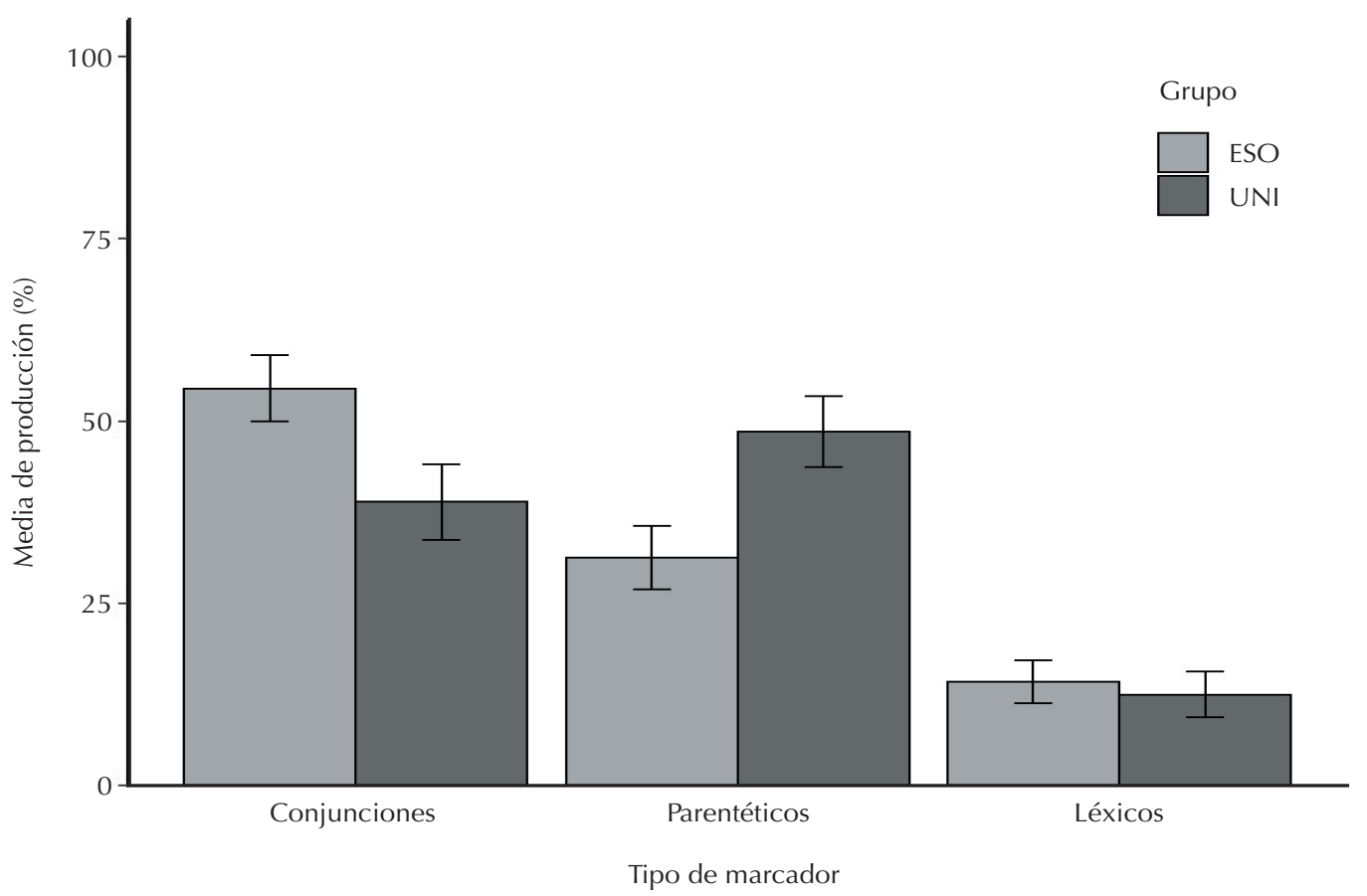

Gráfico 1. Uso de marcadores según forma entre los grupos secundaria y universidad

Fuente: Elaboración propia.

En el segundo análisis se aborda el estudio de los marcadores a partir de la función que cumplen en la construcción del texto. Se computó la proporción de los tres tipos de marcadores según su función (proposicionales, estructurales, modales) sobre el total de marcadores para cada texto. En la tabla 3 se muestran las medidas de centralidad y dispersión de estos marcadores para los grupos de ESO y UNI.

Tabla 3

Distribución de la proporción de marcadores según su función. Secundaria y universidad (media y DT)

Tipo de marcador

\begin{tabular}{|c|c|c|c|c|c|c|}
\hline \multirow[t]{2}{*}{ Nivel educativo } & \multicolumn{2}{|c|}{ Proposicionales } & \multicolumn{2}{|c|}{ Estructurales } & \multicolumn{2}{|c|}{ Modales } \\
\hline & M & DT & M & DT & $\mathrm{M}$ & DT \\
\hline Secundaria (ESO) & 69,25 & 15,67 & 13,41 & 10,97 & 17,33 & 14,55 \\
\hline Universidad (UNI) & 63,69 & 19,60 & 14,59 & 13,32 & 21,70 & 15,34 \\
\hline
\end{tabular}

Fuente: Elaboración propia.

Los marcadores proposicionales son los más frecuentes en los textos de los dos grupos, seguidos de los modales, que ayudan al autor del texto a posicionarse dentro del escrito. Por último, los marcadores estructurales, que organizan la información en el nivel interoracional, son los que presentan una menor frecuencia en ambos grupos. 
Tras comprobar la falta de normalidad en la distribución de los datos y rechazar también el presupuesto de homogeneidad de varianzas $(F(5,378)=3,84, p=0,002)$, se aplicó de nuevo la prueba ANOVA de rangos de Friedman con un diseńo mixto. Los resultados de la prueba no arrojaron efectos principales significativos ni para nivel educativo $(F(1,378)=0,154, p=0,694$, $邓 2<0,01)$ ni para la función de los marcadores $(F(2,18221243)=$ $1,491, p=0,225, \bigotimes 2=0,63)$. Sí resultó significativo, en cambio, el efecto de la interacción entre el nivel educativo y el tipo de marcador según función $(F(2,378)=5,139, p=0,006, \bigotimes 2=0,02)$, aunque con un tamaño del efecto pequeño. Así, observamos que la distribución de proporciones entre los tipos de marcadores según su función difiere significativamente entre los dos grupos. En el gráfico 2 se observa el sentido de la interacción entre los dos factores.

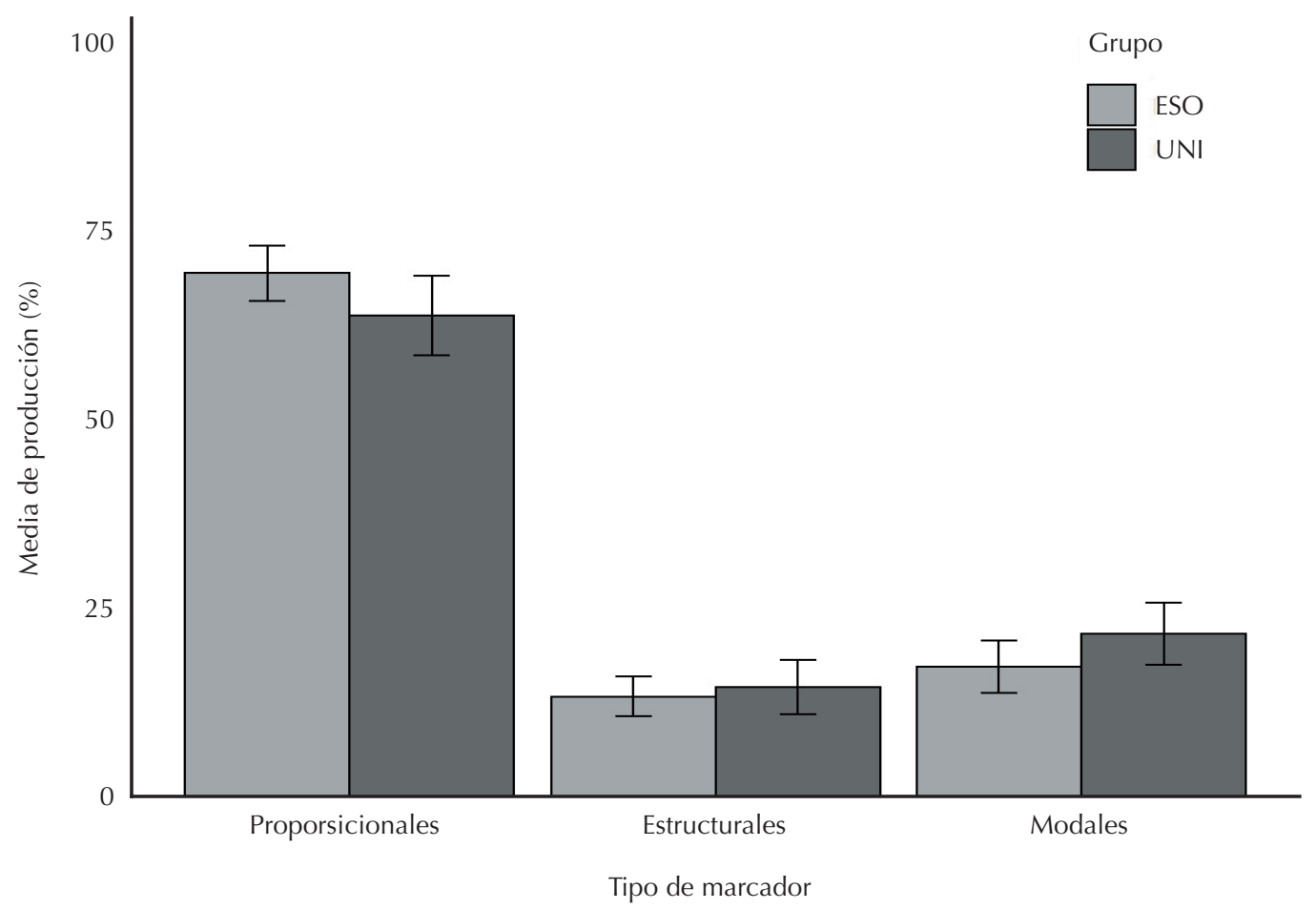

Gráfico 2. Uso de marcadores según función. Secundaria y universidad

Fuente: Elaboración propia.

Para detectar entre qué niveles se encuentran las diferencias dentro de la interacción, se realizó de nuevo una serie de comparaciones por pares aplicando la corrección de Bonferroni sobre el nivel $\bigotimes$ de las pruebas de contraste. En primer lugar, se aplicó un conjunto de comparaciones para contrastar las medias estimadas de proporciones de los dos grupos para cada tipo de función. La proporción de marcadores estructurales resulta estadísticamente equivalente en los dos grupos $(t=-0,56 . p=0,57)$; su uso se ilustra en el ejemplo (4).

(4) Per altra banda n'hi han persones que només volen anar a un altre país amb intencions dolentes, com és en el cas de les persones que porten una bomba o fan un atemptat sense cap motiu [Secundaria, 09]

Trad.: Por otro lado, hay personas que solo quieren ir a otro país con intenciones malas, como es en el caso de las personas que llevan una bomba o hacen un atentado sin ningún motivo. 
La presencia de marcadores proposicionales, que relacionan contenidos conceptuales, como ilustra (5), es significativamente mayor en el grupo de secundaria frente al de universitarios $(t=2,33, p=0,02)$.

(5) No tots som iguals, és per això que la societat no es pugui relacionar(-nos) [Secundaria, 09]

Trad.: No todos somos iguales, es por eso por lo que la sociedad no puede relacionar(nos).

En el caso de los modales, la relación se invierte, es decir, son los textos del grupo UNI los que recogen un mayor número de marcadores que cumplen esta función $(t=-2,13, p=0,03)$. Estos elementos, como vimos, indican las relaciones entre los participantes $y$, de manera crucial en estos textos, el posicionamiento del autor en relación con la información que contiene. Así, el compromiso de los escritores universitarios con su texto parece ser mayor en tanto se posicionan con respecto a aquello que dicen, protegiendo su imagen discursiva por medio de la introducción de fuentes de información como en (6), valorando la credibilidad de esa información como en (7), o evaluándola incluso dando su opinión como en (8).

(6) Segons les dades de Welcome Refugees si tots els països acollissin... [Universidad, 39]

Trad.: Según los datos de Welcome Refugees si todos los países acogiesen...

(7) Aquestes situacions podrien abolir-se al cap d' un mes, desafortunadament no tots els casos acaben així. [Universidad, 39]

Trad.: Estas situaciones podrían abolirse al cabo de un mes, desafortunadamente no todos los casos acaban así.

(8) Crec que, en casos que no són per culpa de guerres i situacions extremes, no és just que nosaltres estiguem treballant i pagant impostos i els immigrants visquin de nosaltres. [Universidad, 64]

Trad.: Creo que, en casos que no son por culpa de guerras y situaciones extremas, no es justo que nosotros estemos trabajando y pagando impuestos y los inmigrantes vivan de nosotros.

El segundo conjunto de comparaciones aborda el análisis de las diferencias entre los tres tipos de función dentro de cada grupo. Si bien el orden de las distribuciones es equivalente en los dos grupos (proposicionales > modales $>$ estructurales), el alcance de las diferencias entre los tipos de función puede ser diferente. En cuanto al grupo ESO, los resultados indican una proporción significativamente mayor de marcadores proposicionales en comparación con la proporción de estructurales $(t=20,63, p<0,001)$ y de modales $(t=16,87, p<0,001)$; en cambio, la comparación entre los dos tipos de marcadores no obligatorios (modales y estructurales) no arroja diferencias significativas $(t=1,64, p=0,305)$ y las comparaciones en los textos de los universitarios generan resultados similares. También aquí la proporción de marcadores proposicionales resulta significativamente superior a la de estructurales $(t=16,60, p<0,001)$ y de modales $(t=12,66, p<0,001)$. No obstante, la proporción de marcas de conexión modales es en este grupo significativamente mayor que la de estructurales $(t=2,73, p=$ $0,022)$, aunque esta diferencia, a pesar de alcanzar nivel de significación estadística, resulta menos contundente teniendo en cuenta el valor del estadístico asociado. Así, en el grupo de universitarios, y a diferencia del grupo de secundaria, los marcadores modales tienen una presencia ligeramente superior a los estructurales. La función estructural es, pues, la menos frecuente en los textos analizados para los dos grupos. 


\section{Discusión}

Para caracterizar cómo tiene lugar la conexión intra e interoracional en los textos analíticos escritos por hablantes de catalán y esbozar un cuadro aproximado del desarrollo que experimentan los estudiantes de secundaria en su tránsito a los estudios universitarios, examinamos las diferencias entre ambos grupos considerando estas marcas de conexión tanto según su forma como según la función discursiva que desempeñan.

En líneas generales, los participantes del grupo de universitarios producen textos significativamente más extensos que los del grupo de secundaria. Este resultado, atestiguado en estudios previos sobre diferentes lenguas, supone evidencia adicional de que la productividad, entendida también en estudios previos como la cantidad de texto producido, es un indicador de desarrollo evolutivo/académico y de calidad textual (Berman \& Nir-Sagiv, 2007; Crossley \& McNamara, 2010; Salas, Llauradó, Castillo, Taulé \& Martí, 2016). La capacidad de producir textos más extensos parece ser prerrequisito para, por ejemplo, desarrollar temas y argumentos de un modo más preciso por medio de oraciones adverbiales que introducen justificaciones y causas, aclaraciones o ejemplos con los que sostener la argumentación (Aparici et al., 2021; González Manzanero \& Rosado, en prensa).

A pesar del aumento de la extensión de los textos con la edad, y contrastando con la tendencia evolutiva hallada en un trabajo paralelo sobre textos en español (Aparici et al., 2021), según nuestros resultados, los textos de secundaria presentan una mayor proporción de marcas de conexión. El reajuste a la baja que se observa en los universitarios está en línea con la idea de que en la ausencia, y no en la profusión, de marcas de cohesión puede residir la coherencia de un texto (Crossley, Weston, McLain Sullivan \& McNamara, 2011); de hecho, en los textos escritos, medios alternativos como las marcas de puntuación realizan las funciones que asumen los marcadores en el discurso oral (Aparici et al., 2014). Por otro lado, en el aprendizaje de determinados recursos lingüísticos se ha reportado con anterioridad una sobreproducción seguida de un ajuste; por ejemplo, la híper conectividad, cuando los niños en edades tempranas, en contextos orales, 'descubren' los conectores (Kupersmitt, 2004; Sebastián \& Slobin, 1994) o se enfrentan más tarde a géneros no narrativos del discurso como el expositivo (Aparici, 2010). En el tipo de texto que nos ocupa, especialmente complejo y de aprendizaje más tardío, este fenómeno de 'reajuste' tendría lugar evolutivamente más tarde, pues en su elaboración deben dedicarse recursos cognitivos a múltiples aspectos que aún no se dominan.

El análisis de las formas utilizadas como marcas de conexión revela la existencia de una suerte de efecto intercambio (trade-off) en función del cual, mientras en secundaria las conjunciones son el recurso más frecuente, el más empleado en los universitarios son los marcadores parentéticos. Vale la pena reparar en la distinta naturaleza de las formas examinadas: si bien las conjunciones (coordinantes y subordinantes) introducen obligatoriamente la oración que encabezan, los marcadores son, por definición, opcionales en su contexto, y su uso constituye una opción retórica por parte del autor (Ravid \& Berman, 2006; Katzenberger \& Cahana-Amitay, 2002). Además, como hemos visto, algunos de estos nexos intraoracionales pueden, en el grupo de secundaria, carecer de su valor original y funcionar como pegamento discursivo, lo que justifica en parte su sobreproducción. Si bien se trata de un fenómeno típico de edades más tempranas y textos orales (Berman, 1996, 2008), nuestros resultados lo atestiguan también en escritos típicos de contextos formales.

Como se ha reportado, la proporción de marcadores léxicos es equivalente en los dos grupos. Los léxicos son marcadores no convencionales, también parentéticos y, por tanto, no obligatorios, que operan a nivel interoracional (Cuenca, 2013). La naturaleza híbrida de esta clase abierta, a medio camino entre un marcador y una cláusula, podría explicar que no constituyan la opción preferida en modalidades o registros formales y merece un análisis pormenorizado en futuros trabajos. De hecho, la identificación y el registro de las formas aparecidas en nuestro corpus constituye una de las aportaciones del trabajo y tiene gran potencial lingüístico y didáctico. En relación con los resultados sobre funciones discursivas, hemos observado en los dos grupos un predominio de marcadores 
proposicionales y un menor uso de marcadores estructurales, así como una presencia ligeramente superior de modales en los universitarios frente a los estudiantes de secundaria, que usan modales y estructurales en la misma medida. La explicación de esta distribución en la frecuencia de las funciones de las marcas de conexión puede encontrarse, por una parte, en la propia configuración de la lengua que hace casi obligatorio el uso de muchos de los marcadores proposicionales para la relación entre contenidos a nivel intraoracional y, por otra, en la inexperiencia relativa de los participantes en la confección de textos que requieren, o al menos favorecen, el uso de marcadores estructurales para demarcar unidades mayores de discurso.

Los marcadores estructurales, que a nivel interoracional demarcan unidades textuales e indican progreso discursivo, son los menos frecuentes en los textos analizados para los dos grupos, a pesar de que los participantes están familiarizados con ellos, puesto que fueron presentados y ejemplificados en el contexto de la secuencia didáctica aplicada. Sabemos, además, que en las aulas de secundaria catalanas se trabaja explícitamente sobre el uso de los conectores del discurso y su rol en la organización de textos analíticos. En este sentido, en la práctica docente parece darse una interesante paradoja, pues, de alguna manera, profesores de todos los niveles educativos cooperan con sus alumnos cuando valoran el contenido del texto por lo que dicen y no por cómo lo dicen: el uso de marcas de conexión, específicamente de marcadores textuales, es un buen ejemplo. En lugar de evaluar los textos en términos de adecuación discursiva, en ocasiones, los docentes deducen del texto lo que no se ha dicho, dando por válidos argumentos formulados erróneamente o haciendo inferencias que van más allá de lo que se dice en el texto, reinterpretando su contenido (Fernández, 2010; Rosado, Aparici \& Perera, 2014). Esta 'complicidad', que en momentos tempranos del desarrollo supone un valioso mecanismo de aprendizaje (por ejemplo, en forma de expansiones y extensiones de los enunciados infantiles por parte de los adultos en un contexto de producción oral; véase Serra, Solé, Serrat, Bel \& Aparici, 2000) no reporta, en realidad, ningún beneficio para alumnos que tienen que aprender a redactar textos académicos.

La expresión de la perspectiva distanciada por parte del autor, propia del discurso expositivo (Tolchinsky, Rosado, Aparici \& Perera, 2005), se materializa en una serie de recursos formales que permiten al autor implicarse o comprometerse más o menos con su texto (Tolchinsky \& Rosado, 2005). Los marcadores modales son uno de estos recursos mediante los cuales el autor enlaza y construye su discurso, argumentando y contraargumentando, modalizando o atenuando su posición. Nuestros resultados indican una evolución en el uso de estos marcadores en los textos de los universitarios quienes, además, los emplean más profusamente que los estructurales.

Aprender a enlazar oraciones por medio de los elementos de conexión que ofrece una lengua dada es una habilidad que se comienza a desarrollar en edades relativamente tempranas (Berman \& Slobin, 1994; Serra et al., 2000). Relacionar de manera competente y eficaz la información contenida en esas oraciones o en unidades de discurso mayores parece constituir, no obstante, un aprendizaje que se prolonga incluso más allá de la adolescencia y que, como se ha observado para otras habilidades discursivas, va íntimamente ligado al género discursivo producido y a las circunstancias en que se produce (Berman, 2008; Tolchinsky, 2004), y está sujeto a un alto grado de variación entre individuos (Aparici et al., 2021). Según nuestros resultados, el uso de conectores modales para ensamblar las piezas que componen un texto permite a su autor posicionarse deliberadamente con respecto a la información transmitida, adoptar una perspectiva discursiva implicada con respecto a dicha información y producir, así, el efecto esperado en el destinatario del texto. Examinar la conexión tanto a nivel oracional como a nivel textual permite detectar problemas que trascienden la habilidad para conectar oraciones y disponer de los recursos para ello, y que afectan a la estructura del texto analítico (Tolchinsky et al., 2021). Además, si consideramos cuál es la función de esa conexión, cuál es la relación que se establece entre sus constituyentes, detectamos problemas que afectan al propósito general del texto y de su autor. En un texto dado, es la presencia o ausencia de información de apoyo y evidencias relevantes, de justificaciones o aclaraciones pertinentes, de explicaciones y definiciones de conceptos específicos, datos de apoyo, o la aparición de falacias provocadas por la incorporación de información 
inadecuada o el establecimiento de relaciones semánticas erróneas lo que nos permitirá identificar si el texto es o no de calidad (Tolchinsky, Aparici \& Rosado, 2017). El tránsito a la universidad no parece poner fin a los problemas que se detectan en secundaria y la escritura de textos analíticos presenta dificultades que persisten más allá de la formación de grado. El uso adecuado de las convenciones utilizadas en este tipo de textos sigue, por ello, siendo una preocupación constante para formadores de todos los niveles y en todas las disciplinas.

Al considerar las limitaciones del estudio y perspectivas de trabajo para el futuro, uno de nuestros propósitos es ampliar el análisis a otros textos de la secuencia didáctica para entender mejor cuáles de los cambios observados son propios del desarrollo evolutivo y cuáles son producto del trabajo pedagógico específico acotado en el tiempo, esto es, de la aplicación de la secuencia.

Identificamos también la conveniencia de realizar una aproximación cualitativa adicional que considere 1) el uso de marcas de conexión en el contexto de los diferentes componentes de la estructura de la argumentación y en relación con su calidad, en la línea de Tolchinsky y sus colegas (2021) y 2) si las formas empleadas por los participantes son adecuadas en términos de ubicación e idoneidad, es decir, si su uso es el esperado en cada contexto, en la línea de Alonso (2019). En el establecimiento de la conexión textual, el empleo de formas o funciones inadecuadas o no canónicas no solo afectará a la calidad del texto en términos de gramaticalidad o estilo, sino que será responsable de la aparición de falacias provocadas por el establecimiento de relaciones semánticas erróneas que adulteran y desvirtúan el propósito inicial del texto, de su autor: persuadir al lector de la validez de su argumento.

Para finalizar, destacamos el potencial didáctico de una propuesta como la nuestra para promover la mejora de la expresión escrita de estudiantes de secundaria y primeros cursos de universidad. La aplicación de este análisis, que parte de problemas y ejemplos reales, se materializa en la confección de una guía de evaluación y autoevaluación de textos analíticos para alumnos y profesores, diseñada para dar respuesta a las dificultades propias de estas etapas educativas (véase Yúfera, Rosado \& Aparici, 2020).

Financiamiento: Esta investigación se ha realizado con la financiación asignada al proyecto competitivo 'Posicionament i Informació-PI: la forma i el fons dels textos analítics en educació secundària i educació superior” (IP: Elisa Rosado). RedICE2018-1920; IDP-Institut de Ciències de l'Educació, Universitat de Barcelona.

El artículo original fue recibido el 25 de enero de 2021

El artículo revisado fue recibido el 22 de junio de 2021

El artículo fue aceptado el 29 de junio de 2021

\section{Referencias}

Alonso-Cortés, M. D., Llamazares, M. T. \& Zapico, L. (2017). La escritura de textos argumentativos: una actividad más satisfactoria gracias a la evaluación compartida. Infancia, Educación y Aprendizaje, 3(2), 350-355. https://doi.org/10.22370/ieya.2017.3.2.747

Alonso, P. (2019). La función de los marcadores discursivos en las variedades hablado y escrito de estudiantes universitarios costarricenses (Tesis doctoral, Universitat de Barcelona). Recuperado de http://hdl.handle.net/2445/151342

Aparici, M. (2010). El desarrollo de la conectividad discursiva en diferentes géneros y modalidades de producción (Tesis doctoral). Universitat de Barcelona, Barcelona, España. 
Aparici, M., Cuberos, R., Salas, N. \& Rosado, E. (2021). Linguistic indicators of quality in analytical texts: developmental changes and sensitivity to pedagogical work. Infancia y Aprendizaje, 44(1), 9-46.

https://doi.org/10.1080/02103702.2020.1848093

Aparici, M. \& García-Palau, M. (2019, septiembre). MUAQ: propuesta de evaluación de la experiencia lingüística y letrada. Comunicación llevada a cabo en el IX Congreso Internacional de Adquisición del Lenguaje AEAL, Madrid, España.

Aparici, M., Rosado, E. \& Salas, N. (2016, julio). On the acquisition of discourse competence in L2 Spanish: the case of discourse markers. Comunicación llevada a cabo en la SIG Writing Conference -15th International Congress of the EARLI Special Interest Group on Writing. Liverpool Hope University, Liverpool, Inglaterra.

Aparici, M., Rosado, E. \& Oliver, A. (2014, agosto). The role of discourse markers in developing text construction. Comunicación llevada a cabo en el SIG Writing Conference -14th International Congress of the EARLI Special Interest Group on Writing. Universidad de Ámsterdam, Ámsterdam, Países Bajos.

Bañales, F. G., Vega L. N., Araujo A. N., Reyna, V. A. \& Rodríguez, Z. B. (2015). La enseńanza de la argumentación escrita en la universidad: una experiencia de intervención con estudiantes de lingüística aplicada. Revista Mexicana de Investigación Educativa, 20(66), 879-910.

Berman, R. A. (1988). On the ability to relate events in narratives. Discourse Processes, 11(4), 469-497. https://doi.org/10.1080/01638538809544714

Berman, R. A. (1996). Form and function in developing narrative abilities: The case of 'and'. En D. Slobin, J. Gerhardt, A. Kyratzis \& J. Guo (Eds.), Social Interaction, Social Context, and Language: Essays in Honor of Susan ErvinTripp (pp. 243-268). Hillsdale, NJ: LEA.

Berman, R. A. (2008). The psycholinguistics of developing text construction. Journal of Child Language, 35(4), $735-771$. https://doi.org/10.1017/S0305000908008787

Berman, R.A. \& Nir-Sagiv, B. (2007). Comparing Narrative and Expository Text Construction Across Adolescence: A Developmental Paradox. Discourse Processes, 43(2), 79-120. https://doi.org/10.1080/01638530709336894

Berman R. A. \& Slobin, D. I. (1994). Relating events in narrative: A cross-lingüistic developmental study. Hillsdale, NJ: Lawrence Erlbaum \& Associates.

Biber, D. \& Gray, B. (2010). Challenging stereotypes about academic writing: Complexity, elaboration, explicitness. Journal of English for Academic Purposes, 9(1), 2-20. https://doi.org/10.1016/j.jeap.2010.01.001

Biber, D., Gray, B., Staples, S. \& Egbert, J. (2020). Investigating grammatical complexity in L2 English writing research: Linguistic description versus predictive measurement. Journal of English for Academic Purposes, 46, 100869. https://doi.org/10.1016/j.jeap.2020.100869

Brandes, G. \& Ravid, D. (2019). The development of adverbial clause functions in Hebrew narrative and expository writing across adolescence. Written Language \& Literacy, 22(1),130-159. https://doi.org/10.1075/wll.00022.bra

Carlino, P. (2005). Escribir, leer y aprender en la Universidad. Una introducción a la alfabetización académica. Buenos Aires, Argentina: Fondo de Cultura Académica.

Castelló, M. (2007). Escribir y comunicarse en contextos científicos y académicos: Conocimientos y estrategias. Barcelona, España: Graó.

Choi, I. (2007). How and when do children acquire the use of discourse markers? En Proceedings of CamLing 2007, The Fifth Cambridge Postgraduate Conference in Language Research (pp. 40-47). Cambridge, Inglaterra: Cambridge University Press.

Crossley, S. A. \& McNamara, D. S. (2010). Cohesion, coherence, and expert evaluations of writing proficiency. En S. Ohlsson \& R. Catrambone (Eds.), Proceedings of the 32nd annual conference of the cognitive science society (pp. 984-989). Cognitive Science Society.

Crossley, S. A., Weston, J., McLain Sullivan, S. T. \& McNamara, D. S. (2011). The development of writing proficiency as a function of grade level: A linguistic analysis. Written Communication, 28(3), 282-311. https://doi.org/10.1177/0741088311410188

Cuberos, R. (2019). Indicadores léxicos de calidad textual en español nativo y no nativo (Tesis doctoral. Universitat de Barcelona). Recuperado de http://hdl.handle.net/2445/178686

Cuenca, M. J. (2006). Connexió i connectors. Vic, España: Eumo. 
Cuenca, M. J. (2013). The fuzzy boundaries between discourse marking and modal marking. En L. Degand, B. Cornillie \& P. Pietrandrea (Eds.), Discourse Markers and Modal Particles. Categorization and description (pp. 181-216). Ámsterdam, Países Bajos: John Benjamins.

Cuenca, M.J. (2017). Connectors gramaticals i connectors lèxics en la construcció discursiva del debat parlamentari. Zeitschrift für Katalanistik, 30, 99-121.

Driver, R., Newton, P. \& Osborne, J. (2000). Establishing the norms of scientific argumentation in classrooms. Science Education, 84(3), 287 - 312. https://doi.org/10.1002/(SICI)1098-237X(200005)84:3<287::AID-SCE1>3.0.CO;2-A

Fernández, S. (2010). La competencia discursiva. Monografías marcoele, 11, 351-383.

Gavari, E. I. \& Tenca, P. (2017). La evolución histórica de los Centros de Escritura Académica. Revista de Educación, 378, 9-29.

Givón, T. (1992). The grammar of referential coherence as mental processing instructions. Linguistics 30, 5-55. https://doi.org/10.1515/ling.1992.30.1.5

González Manzanero, R. \& Rosado, E. (en prensa). Conectividad causal en el discurso: hacia una caracterización global de las construcciones causales en espańol. Signos, 108(55).

Gras, P., Galiana, P. \& Rosado, E. (2020). Modal and discourse marking in L1 \& L2 Spanish: A comparative analysis of oral narratives. Corpus Pragmatics, 5, 63-94. https://doi.org/10.1007/s41701-020-00081-1

Hyland, K. (2002). Teaching and researching writing. Harlow, Inglaterra: Longman/Pearson Education Limited.

Katzenberger, I. \& Cahana-Amitay, D. (2002). Segmentation marking in text production. Linguistics 40(6), 1161-1184. https://doi.org/10.1515/ling.2002.043

Konietschke, F., Placzek, M., Schaarschmidt, F. \& Hothorn, L. (2015). nparcomp: An R Software Package for Nonparametric Multiple Comparisons and Simultaneous Confidence Intervals. Journal of Statistical Software, 64(9), 1-17. https://doi.org/10.18637/jss.v064.i09

Kupersmitt, J. (2004). Form-Function Relations in Spanish Narratives. A comparative Study of Bilingual and Monolingual Speakers. En S. Strömqvist \& L. Verhoeven (Eds.), Relating events in narrative: Typological and Contextual Perspectives (vol. II) (pp. 395-434). Hillsdale, NJ: LEA.

MacWhinney, B. (2000). The CHILDES Project: Tools for Analyzing Talk(3rd ed.). Mahwah, NJ: Lawrence Erlbaum \& Associates.

Martín Zorraquino, M. A. \& Portolés, J. (1999). Los marcadores del discurso. En I. Bosque \& V. Demonte (Eds.), Gramática Descriptiva de la Lengua Española (Vol. 3, pp. pp. 4051-4213). Madrid, España: Espasa Calpe.

Montolío, E. (2001). Conectores de la lengua escrita. Barcelona, España: Ariel.

Ravid, D. \& Berman, R.A. (2006). Information Density in the Development of Spoken and Written Narratives in English and Hebrew. Discourse Processes, 41(2), 117-149. https://doi.org/10.1207/s15326950dp4102_2

Rosado, E., Aparici, M., Horcada, P., Cuberos, R. \& Salas, N. (2017, julio). Discourse markers and syntactic connectives in L2 Spanish: a developmental path across discourse genres and modalities of production. Póster presentado en el XIVth International Congress for the Study of Child Language. Universidad de Lyon-CNRS, Lyon, Francia.

Rosado, E., Aparici, M. \& Perera, J. (2014). De la competencia discursiva en español L2 o de cómo adaptarse a las circunstancias. Cultura y Educación, 26(1), 71-102. https://doi.org/10.1080/11356405.2014.908671

Ruiz Bikandi, U. \& Tusón, A. (2002). Explicar y argumentar. Textos de Didáctica de la Lengua y la Literatura, (29), 5-10.

Salas, N., Llauradó, A., Castillo, C., Taulé, M. \& Martí, M. A. (2016). Linguistic correlates of text quality from childhood to adulthood. En J. Perera, M. Aparici, E. Rosado \& N. Salas (Eds.), Written and spoken language development across the lifespan. Essays in honour of Liliana Tolchinsky (pp. 307-326). Springer.

Schleppegrell, M. J. (2004). The language of schooling. A functional linguistics perspective. Lawrence Erlbaum.

Sebastián, E. \& Slobin, D.I. (1994). Development of linguistic forms: Spanish. En R.A. Berman \& D. I. Slobin (Rds.), Relating events in narrative: A crosslinguistic developmental study (pp. 239-284). Hillsdale, NJ: LEA.

Serra, M., Solé, M.R., Serrat, E., Bel, A. \& Aparici, M. (2000). La adquisición del lenguaje. Barcelona, España: Ariel.

Snow, C. E. \& Uccelli, P. (2009). The challenge of academic language. En D. R. Olson \& N. Torrance (Eds.), The Cambridge Handbook of Literacy (pp. 112-133). Cambridge, Inglaterra: Cambridge University Press. 
Tolchinsky, L. (2004). The nature and scope of later language development. En R. A. Berman (ed.), Language Development across Childhood and Adolescence (pp. 233-248). Ámsterdam, Países Bajos: John Benjamins.

Tolchinsky, L., Aparici, M. \& Rosado, E. (2017). Escribir para pensar y persuadir. Textos de Didáctica de la Lengua y la Literatura, (76), 14-21.

Tolchinsky, L., Aparici, M. \& Salas, N. (2011, julio). The development of syntactic depth in Spanish narratives. Comunicación llevada a cabo en el XII International Congress for the Study of Child Language. Université de Montreal, Montreal, Canadá.

Tolchinsky, L., Aparici, M. \& Vilar, H. (2021). Macro- and micro-developmental changes in analytical writing of bilinguals from elementary to higher education. International Journal of Bilingual Education and Bilingualism. https://doi.org/10.1080/13670050.2021.1923643

Tolchinsky, L. \& Rosado, E. (2005). The effect of literacy, text type, and modality on the use of grammatical means for agency alternation in Spanish. Journal of Pragmatics, 37, 209-237. https://doi.org/10.1016/j.pragma.2004.08.008

Tolchinsky, L., Rosado, E., Aparici, M. \& Perera, J. (2005). Becoming proficient educated users of language. En D. Ravid \& H. Bat-Zeev Shyldkrot (Eds.), Perspectives on Language and Language Development. Essays in Honor of Ruth A. Berman (pp. 375-389). Dordrecht, Países Bajos: Kluwer.

Toulmin, S. (2002). The uses of argument. Cambrige, Inglaterra: Cambridge University Press.

Uccelli, P., Barr, C., D., Dobbs, C. L., Phillips Galloway, E., Meneses, A. \& Sánchez, E. (2015). Core Academic Language Skills (CALS): An expanded operational construct and a novel instrument to chart school-relevant language proficiency in pre-adolescent and adolescent learners. Applied Psycholinguistics, 36(5), 1077-1109. https://doi.org/10.1017/S014271641400006X

Uccelli, P., Deng, Z., Phillips Galloway, E. \& Qin, W. (2019). The role of language skills in midadolescents' science summaries. Journal of Literacy Research, 51(3), 357-380. https://doi.org/10.1177/1086296X19860206

Uccelli, P., Dobbs, C. \& Scott, J. (2012). Mastering academic language: Organization and stance in the persuasive writing of high school students. Written Communication, 30(1), 36-62. https://doi.org/10.1177/0741088312469013

Uccelli, P., Dobbs, C. \& Scott, J. (2013). Mastering Academic Language. Written Communication, 30(1), 36-62. https://doi.org/10.1177/0741088312469013

Wobbrock, J., Findlater, L., Gergle, D. \& Higgins, J. (2011). The aligned rank transform for nonparametric factorial analyses using only anova procedures. En D. Tan, G. Fitzpatrick, C. Gutwin, B. Begole y W. Kellogg (Eds.), Proceedings of the SIGCHI Conference on Human Factors in Computing Systems (pp. 143-146). https://doi.org/10.1145/1978942.1978963

Yúfera, I., Rosado, E. \& Aparici, M. (2020, julio). Does form always follow function? Connecting information in academic writing. Comunicación presentada en el Fourth International Conference on Academic Writing (IFAW 2020). Blurring the Lines: Academic, Professional, and Popular Writing. Universidad de Tel Aviv, Tel Aviv, Israel. 